\title{
DISEÑO DE UN MODELO MATEMÁTICO FORMULADO A PARTIR DE VARIABLES SOCIOECONÓMICAS PARA ESTIMAR LA GENERACIÓN DE RESIDUOS SÓLIDOS URBANOS EN EL DISTRITO DE HUACHO.
}

\author{
DESIGN OF A MATHEMATICAL MODEL FORMULATED FROM SOCIOECONOMIC VARIABLES TO \\ ESTIMATE THE GENERATION OF SOLID URBAN WASTE IN THE HUACHO DISTRICT.
}

\author{
Juan Manuel Ipanaque Roña ${ }^{1}$, Ronald Fernando Rodríguez Espinoza ${ }^{1}$, Berardo Beder Ruiz Sanchez ${ }^{1}$ \\ Alberto I. Sánchez Guzman ${ }^{1}$ José Vicente Nunja García ${ }^{1}$ Baleriano Alfonzo Díaz Guzmán
}

\section{RESUMEN}

El Objetivo, diseñar un modelo matemático a partir de las variables socioeconómicas que correlacione y estimar confiablemente la generación de los Residuos Sólidos Urbanos usando la regresión múltiple como técnica estadística. En la Metodología, se consideraron 61 muestras para cada estrato socioeconómico, obtenidas a partir de un muestreo aleatorio estratificado, con un total de 122 muestras. Resultados, la producción total de los residuos sólidos del distrito de Huacho, fue de $35770 \mathrm{~kg} /$ día, generando un ingreso diario de S/. 5241.27 ; la producción per cápita promedio de $0.4 \mathrm{~kg} / \mathrm{hab} /$ día y la densidad de $273.20 \mathrm{~kg} / \mathrm{m} 3$. La hipótesis, afirma que el modelo matemático existe una correlación entre las variables socioeconómicas y la generación de residuos sólidos municipales de $40.8 \%$ y un valor de significancia 0.00 ; el modelo de regresión elegida para los encuestados es válido, con un nivel de significancia del 0.05 . Se concluye que, a través de un modelo matemático de regresión múltiple, se obtiene una estimación confiable de la generación de residuos sólidos municipales.

Palabras clave: Modelo matemático; variables; generación de residuos sólidos.

\begin{abstract}
The objective is to design a mathematical model based on the socioeconomic variables that correlate and reliably estimate the generation of Urban Solid Waste using multiple regression as a statistical technique. In the Methodology, 61 samples were considered for each socioeconomic stratum, obtained from a stratified random sampling, with a total of 122 samples. Results, the total production of solid waste from the district of Huacho was $35,770 \mathrm{~kg} /$ day, generating a daily income of S /. 5 241.27; the average per capita production of $0.4 \mathrm{~kg} / \mathrm{inhab} /$ day and the density of $273.20 \mathrm{~kg} / \mathrm{m} 3$. The hypothesis, affirms that the mathematical model exists a correlation between the socioeconomic variables and the generation of municipal solid waste of $40.8 \%$ and a value of significance 0.00 ; The regression model chosen for the respondents is valid, with a level of significance of 0.05. It is concluded that, through a mathematical model of multiple regression, a reliable estimate of the generation of municipal solid waste is obtained.
\end{abstract}

Key words: Mathematical model; variables; generation of solid waste

${ }^{1}$ Universidad Nacional José Faustino Sánchez Carrión de Lima (Lima - Perú). Email:vinvestigación@unjfsc.edu.pe 


\section{INTRODUCCIÓN}

En 1990, los países por la Organización para la Cooperación y el Desarrollo Económico (OCDE), produjeron alrededor de 9 mil millones de toneladas de desechos. Su distribución por origen fue: 420 millones de toneladas de desechos urbanos, 1500 millones de desechos industriales, entre ellos 300 millones de desechos peligrosos, y más de 7 mil millones de otros desechos originados por las actividades agrícolas, mineras, producción de energía, demoliciones y sistemas de saneamiento. La magnitud de los problemas es evidente, por ejemplo, los desechos industriales en 1990 crecieron un 50\% respecto al año 1980.

Cada habitante latinoamericano produce casi un kilo de basura/día. América Latina y el Caribe, generan a diario 436000 toneladas de residuos sólidos urbanos (0.93 kilos per cápita), casi $60 \%$ más que 1995, que se arrojaban 275000 toneladas (0.75 kilos por persona).Según el INEI, XI: Censo Nacional de Población del 2007, cuenta con 28220764 habitantes. Las ciudades más pobladas se ubican en la costa, como resultado de la migración desde la zona rurales, agudizándose desde la década de 1950. El departamento de Lima, se concentra 9 millones de habitantes ( $29 \%$ de la población total), con una tasa de crecimiento $2 \%$ anual. El aumento poblacional y consumo que se registra entre sus habitantes, se ha visto reflejado con el incremento de los residuos sólidos que se producen en las casas, mercados, centros comerciales y hasta en las industrias.

El reporte de la Dirección General de Salud Ambiental (Digesa) del Ministerio de Salud precisa que Lima, genera diariamente unas 6750 toneladas de basura, es decir que cada limeño produce al día 0.85 kilogramos de desechos. El 80\% de esta basura es recogida por las empresas concesionarias y municipales, mientras que el resto se queda regado en calles, parques, plazas y jardines, descomponiéndose y contaminando el ambiente. Por lo tanto, los residuos que genera la sociedad urbana están directamente relacionados con las actividades de la sociedad y los insumos que consume. La generación, composición, recolección, tratamiento y disposición final de los residuos, se relacionan con las actividades e insumos, y se clasifica para estudio en residuos sólidos peligrosos, industriales y municipales.

La Ley General de Residuos Sólidos N ${ }^{\mathrm{a}} 27314$ precisa las responsabilidades de las Autoridades Municipales, a nivel provincial como distrital. El ámbito de competencia de ambas comprende los residuos sólidos municipales de origen domiciliario, comercial y de aquellas actividades que generen residuos similares a estos, en todo el ámbito de su jurisdicción. La Municipalidad Provincial tiene a su cargo la gestión integral de los residuos en su jurisdicción, y debe coordinar con las Municipalidades Distritales y Centros Poblados menores. El articulo IV del Título Preliminar de la Ley Orgánica de Municipalidades, establece que las Municipalidades Distritales son responsables de la limpieza de vías, espacios y monumentos públicos en su jurisdicción, así como de la prestación de los servicios de recolección y transporte de los residuos sólidos municipales, y deberán ser conducidos a la planta de tratamiento, transferencia o al lugar de disposición final autorizado por la municipalidad.

La falta de implementación de un sistema integral que involucre los tres niveles de Gobierno: Central, Regional y Local. La generación de los Residuos Sólidos Municipales, es uno de los elementos de la Gestión Integral de Residuos Sólidos Municipales, esta cantidad de residuos que genera una persona en un día, también se le conoce como generación por persona o per cápita. Un correcto manejo de los residuos sólidos, desde la generación hasta la disposición final, permite realizar una adecuada gestión integral de residuos sólidos municipales. En una ciudad grande o pequeña se debe conocer la cantidad de residuos sólidos a recoger y evaluar sus características, composición, densidad, humedad, solido volátil y otros, permitiendo un buen diseño de los sistemas para el manejo de los residuos sólidos (Digesa, 1996).

En el ámbito nacional, la generación diaria per cápita de residuos sólidos pasó de 0.71 para el 2001 a 1.08 en el 2007 (kg/hab/día); y Lima es la ciudad que genera más de la tercera parte de los residuos sólidos a nivel nacional. E1 68\% del total de residuos municipales son domiciliarios, mientras que $13 \%$ proviene de los mercados y el resto de establecimientos comerciales, de salud, parques y jardines entre otros (Minam, 2008).

Según Armillas, F. et al (2009), los distritos de la provincia de Huaura, el 37\% de los hogares cuentan con el servicio municipal de recojo de basura y $39,2 \%$ adicionales cada dos o tres días; y de forma esporádica, una vez a la semana o de vez en cuando $22,9 \%$. Además, de los residuos sólidos generados en los domicilios, en el distrito de Huacho existen 04 mercados de consumo: Mercado Centenario, Mercado Modelo, Mercado San Bartolomé y 
Mercado Puquio Cano, donde los principales residuos generados son de características orgánico.

El municipio de Distrito de Huacho, ha realizado estudios precisos en lo referente a la generación de RSM, la ppc para el año 2009 fue 0.424 y para el 2014 una generación $0.611 \mathrm{~kg} / \mathrm{hab} /$ día por persona como promedio, se considera que existe 58543 habitantes, obteniendo 35.77 ton/día de RSD.

La provincia de Huaura, existe un botadero llamado "Paraíso", donde se deposita más de 30 años los desperdicios de los 05 distritos de la provincia (Huacho, Hualmay, Huaura, Santa María y Carquin). Este botadero, se ubica en el kilómetro 143 de la Panamericana Sur; visible desde dicha ruta de transporte restando competitividad turística al norte chico (Arnillas, F. et al, 2009).

El objeto de estudio de esta investigación, se relaciona con la generación de residuos sólidos urbanos y como caso particular, aquellos residuos generados en el distrito de Huacho. Este sujeto de estudio se relaciona con el modelo matemático, y permite conocer la relación existente, entre la sociedad y la naturaleza, para predecir bajo ciertas condiciones, la generación de residuos sólidos urbanos. Tener presente, que la modelación de la generación de residuos sólidos, al igual que cualquier parámetro de carácter social depende, de las variables socioeconómicas, estaciones del año y de la cultura de quien genera los residuos sólidos. Dadas las condiciones que antecede, un modelo matemático sencillo que permita conocer con un simple cálculo una estimación confiable útil en la toma de decisiones.

La generación de residuos sólidos urbanos del distrito de Huacho se puede estimar confiablemente de forma factible y económica aplicando un modelo matemático formulado a partir de variables socioeconómicas: del estrato medio y del estrato bajo.

\section{Definiciones Conceptuales}

a.- Sistema: Es un conjunto de partes que interaccionan, como si fuera una caja, dentro de la cual se puede mirar, y como se relacionan con otros sistemas. (Tracheta, 2005).

b.-Universo: En general, el universo es la totalidad de elementos o características que conforman el ámbito de un estudio o investigación. Materia, energía, espacio y tiempo, todo aquello que existe, incluso el sistema y es infinito c.- Modelo: Es una forma de representa al sistema, el modelo es un sistema en donde solo hay flujo de información que representa una realidad concreta, los modelos pueden ser complicado o simples.

d.- Vivienda: Espacio delimitado normalmente por paredes y techos de cualquier material, con entrada independiente, que se utiliza para vivir, esto es, dormir, preparar los alimentos, comer y protegerse del ambiente.

e.- Producto bruto interno (PIB): Producción total para uso final de bienes y servicios de una economía, realizada tanto por residentes como por no residentes.

f.- Unidad Económica Total: Es unidad que bajo una sola entidad propietaria o controladora se dedica a alguna de las actividades económicas.

g.- Producción Bruta Total: Es el valor de los bienes y servicios producidos por las unidades económicas durante el año del censo como resultado del ejercicio de sus actividades.

h.- Personal Ocupado: Suma de promedios del personal ocupado total remunerado y no remunerado de las unidades económicas. El promedio de cada unidad económica se calcula dividiendo entre dos la suma del personal ocupado total que se obtuvo en dos fechas distintas.

i.- Energía: El término energía (del griego, actividad, operación; "fuerza de acción" o "fuerza trabajando") tiene diversas acepciones y definiciones, relacionadas con la idea de una capacidad para obrar, transformar o poner en movimiento

\section{OBJETIVO}

Determinar la generación de residuos sólidos urbanos en el distrito de huacho, en forma factible y económica, con el diseño de un modelo matemático formulado a partir de las variables socioeconómicas, del estrato medio y del estrato bajo.

\section{MATERIALES Y MÉTODOS}

El estudio de los residuos sólidos, se desarrolló en el distrito de Huacho, provincia de Huaura, departamento Lima. El Diseño de Investigación, es de tipo descriptivo correlacionar, diseño no experimental de corte transversal o transaccional. La investigación descriptiva comprende la descripción, registro, análisis e interpretación de las condiciones existentes en el momento.

La Población del Distrito de Huacho, está 
compuesta por 58543 habitantes (Anexo $\mathrm{N}^{\mathrm{o}} 01$ ), calculándose el número de viviendas de 16307 . La determinación de la muestra, generación y características física de los RSU, varía en función a los hábitos de consumo y nivel de vida de la población (estratos socioeconómicos), seleccionándose dos estratos socioeconómicos para huacho, tomándose 122 muestras (Rodríguez E. 2009$) .61$ corresponde al estrato socioeconómico, medio - medio bajo, una población de 37819 habitantes y 10534 viviendas. Y 61 muestra para el estrato bajo, una población de 20724 habitantes y 5854 viviendas.

\section{Método}

Numero de Vivienda para el año 2014: anexo 01 Fuente de información, Municipalidad de Huaura año 2014. La población se determino por estrato socioeconómico: Medio-Medio Bajo, se consideró a la Urb. Lever Pacocha (64.6\%), 61.12 viviendas y el Estrato bajo, al sector la Manchuria (35.4\%), 60.83 viviendas de un total de 122 viviendas, (Rodríguez E. 2009). Distribuyéndose de la siguiente manera 61 vivienda para cada estrato. ReferenciaAnexo 8.

$$
n=\frac{V^{9}}{\left(\frac{E}{1,96}\right)^{2}+\frac{V^{2}}{N}}
$$

Dónde:

$n$ : Número de viviendas a probar aleatoriamente.

$V$ : Desviación estándar de la producción per Cápita de las viviendas.

$E$ :Error permisible en la estimación de la producción per Cápita.

$N$ : Número total de viviendas del estrato en cuestión.

Flores y Villafuerte (2003) utilizar los valores, confiabilidad de 95\%:

\section{Error permisible $\quad: 50 \mathrm{~g} / \mathrm{hab} / \mathrm{dí}$}

Desviación estándar: 200 g/hab/día.

Técnicas de Recolección de Datos: encuestas y además un díptico, y Sistema de Generación de RSU: para validar el modelo se usó dos datos: índice de generación per cápita (gpc) de 0.611 $\mathrm{kg} / \mathrm{hab} /$ día Anexo 01 Fuente, Municipalidad de Huaura año 2014. El otro atreves de encuetas y cuestionarios, Rodríguez E. 2009. Se tomaron encuetas.

Determinación de las zonas de limpieza: La Municipalidad Provincial de Huaura, está a cargo de los servicios de limpieza pública, parque y jardines,

Determinación del ingreso diario: Multiplicando la producción per cápita por la población total del distrito, obtenemos la producción total de residuos sólidos $35770 \mathrm{~kg} /$ día.

\section{RESULTADOS}

La producción total de los residuos sólidos del distrito de Huacho, fue de $35770 \mathrm{~kg} /$ día, generando un ingreso diario de S/. 5 241.27 La producción per cápita promedio de $0.4 \mathrm{~kg} / \mathrm{hab} /$ día y la densidad de $273.20 \mathrm{~kg} / \mathrm{m} 3$.

Tabla 1. Ingreso Diario aproximado de cada Segregado

\begin{tabular}{|c|c|c|c|c|}
\hline № & Materiales segregados & Cantidad (Kg) & $\begin{array}{l}\text { Precio } \\
\mathrm{S} / . \mathrm{Kg}\end{array}$ & $\begin{array}{c}\text { Ingreso } \\
\mathrm{S} / \mathrm{Kg}\end{array}$ \\
\hline & Inorgánicos y/o no putrescibles & 11088.7 & & 3180.56 \\
\hline 1 & Aluminio & 9.66 & 3,7 & 35.73 \\
\hline 2 & Cartón & 892.10 & 0,2 & 178.42 \\
\hline 3 & Cuero & 103.02 & 0 & 0.00 \\
\hline 4 & Huesos & 0 & 0 & 0.00 \\
\hline 5 & Jebes y sintéticos & 409.21 & 0 & 0.00 \\
\hline 6 & Latas y latones & 786.94 & 0,35 & 276.48 \\
\hline 7 & Madera & 11.80 & 0 & 0.00 \\
\hline 8 & Metal ferroso & 0 & 0,3 & 0.00 \\
\hline 9 & Pañales descartables & 2422.34 & 0 & 0.00 \\
\hline 10 & Papel blanco & 622.76 & 0,5 & 311.38 \\
\hline 11 & Papel mixto o periódico & 1415.31 & 0,1 & 141.53 \\
\hline 12 & Pilas y baterías & 23.25 & 0 & 0.0 \\
\hline 13 & Plástico liviano & 1973.07 & 0,6 & 1183.84 \\
\hline 14 & Plástico pesado o rígido & 1049.13 & 0,9 & 944.22 \\
\hline 15 & Tetra pak & 309.77 & 0,05 & 15.49 \\
\hline 16 & Trapos & 199.60 & 0 & 0.0 \\
\hline \multirow[t]{5}{*}{17} & Vidrio & 861.34 & 0,15 & 129.20 \\
\hline & Orgánicos y/o putrescibles & 20342.76 & 0,1 & 2034.28 \\
\hline & Inertes & 4311.72 & 0 & 0.0 \\
\hline & Residuos peligrosos & 26.83 & 0 & 0.0 \\
\hline & Masa total & 35770.00 & & 5241.27 \\
\hline
\end{tabular}

Tabla 2. GPC del Distrito de Huacho

\begin{tabular}{lllll}
\hline Estratos & GPC & Población 2013 & Muestras & $\begin{array}{l}\text { GPC Distrital } \\
\text { Kg/Hab./Día }\end{array}$ \\
\hline Alto & & 0 & 0 & 00.40 \\
Medio & 0.45 & 37819 & 61 \\
Bajo & 0.35 & 20724 & 61 \\
Totales & 0.40 & 58543 & 122 \\
\hline
\end{tabular}


Tabla 3. Densidad de los residuos de Huacho

\begin{tabular}{cccc}
\hline \multirow{2}{*}{ Zonas Promediadas } & \multicolumn{3}{c}{ Densidad (Kg/m3) } \\
\cline { 2 - 4 } & Sin Compactar & Compactada & Promedio \\
\hline Alta & 0 & 0 & 0 \\
Media & 230,865 & 295,223 & 263,044 \\
Baja & 249,808 & 316,910 & 283,359 \\
\hline
\end{tabular}

Se observa que la mayor densidad se da en nivel socioeconómico bajo, se debería a su composición física de residuos del mencionado estrato predominando los residuos orgánicos e inorgánicos. La diferencia de densidad entre niveles socioeconómicos medio y bajo está relacionada con los hábitos de consumo de la población, la misma que se puede analizar en las Tablas.

La hipótesis, afirma que el modelo matemático existe una correlación entre las variables socioeconómicas y la generación de residuos sólidos municipales de $40.8 \%$ y un valor de significancia 0.00; indicando que el modelo de regresión elegida para los encuestados es válido, con un nivel de significancia del 0.05

Se realizaron tres correlaciones con los valores determinados donde la variable dependiente, Y: es la generación de residuos sólidos y las variables independientes son las socioeconómicas representados por el X1: Ingreso económico, X2: consumo de energía y X3: número de habitantes.

Nuestro análisis ha utilizado el nivel de significación del $0.05 \mathrm{El}$ modelo de regresión múltiple presenta el siguiente modelo

$$
\mathrm{Y}=\beta+\beta \quad \mathrm{X}_{i} \hat{1}^{+} \beta \mathrm{X}_{i}+\mathrm{E}
$$

Asimismo, se utilizó el diseño estadístico de correlación de Pearson, que es un índice estadísticos que mide la relación lineal entre dos variables cuantitativas, el cual el valor del índice de correlación varía en el intervalo

Tabla 4. $R$ cuadrado entre Variables socioeconómicas y Generación de residuos

\begin{tabular}{ccccc}
\hline Modelo & $\mathrm{R}$ & R cuadrado & $\begin{array}{c}\text { R cuadrado } \\
\text { corregida }\end{array}$ & $\begin{array}{c}\text { Error típ. de } \\
\text { la } \\
\text { estimación }\end{array}$ \\
\hline 1 & $.696^{\mathrm{a}}$ & 0.484 & 0.471 & 0.179223 \\
\hline $\begin{array}{l}\text { a.Variables predictoras: (Constante), número de habitantes, consumo de } \\
\text { energía, ingreso económico }\end{array}$
\end{tabular}

Al realizar el análisis por Regresión múltiple entre las variables socioeconómicas y la generación de residuos, se observa en la tabla 4.20 que el $\mathrm{R}$ cuadrado corregida es de 0.484 esto significa estadísticamente que $40.8 \%$ de las variaciones de la generación de solidos pueden ser explicadas a partir de las variables socioeconómicas.

De los resultados obtenidos en la tabla 4.22 tenemos:

Tabla 5. Anova entre variables socioecómicas y generación de residuos

\begin{tabular}{|c|c|c|c|c|c|c|}
\hline & Modelo & $\begin{array}{c}\text { Suma de } \\
\text { cuadrados }\end{array}$ & gl & $\begin{array}{c}\text { Media } \\
\text { cuadrática }\end{array}$ & $\mathbf{F}$ & Sig. \\
\hline \multirow{3}{*}{1} & Regresión & 3.561 & 3 & 1.187 & 36.957 & $.000^{\phi}$ \\
\hline & Residual & 3.79 & 118 & 0.032 & & \\
\hline & Total & 7.352 & 121 & & & \\
\hline
\end{tabular}

Anova nos muestra un Sig. 0.000, resultado que indica que el con un nivel de significancia del 0.05 . modelo de regresión elegida para los encuestados es válido,

Tabla 6. Coeficientes relacionados entre Variables socioeconómicas y Generación de residuos.

\begin{tabular}{|c|c|c|c|c|c|c|}
\hline \multirow[t]{2}{*}{ Modelo } & & \multicolumn{2}{|c|}{$\begin{array}{l}\text { Coeficientes no } \\
\text { estandarizados }\end{array}$} & \multirow{2}{*}{$\begin{array}{c}\text { Coeficientes } \\
\text { tipificados } \\
\text { Beta }\end{array}$} & \multirow[t]{2}{*}{$\mathrm{t}$} & \multirow[t]{2}{*}{ Sig. } \\
\hline & & B & Error típ. & & & \\
\hline \multirow{4}{*}{1} & (Constante) & 0.535 & 0.065 & & 8.181 & 0 \\
\hline & corriente & 0.003 & 0 & 0.545 & 6.981 & 0 \\
\hline & sueldo & $9.96 \mathrm{E}-05$ & 0 & 0.166 & 2.086 & 0.039 \\
\hline & hab & -0.018 & 0.009 & -0.139 & -2.011 & 0.047 \\
\hline
\end{tabular}

a. Variable dependiente: ppc 
$Y=0.535+0.003 X_{1}+0.00009956 X_{2}-0.018 X_{3}$

Se observa en la tabla 4.22 que los coeficientes de significancia dieron de resultado para el número de habitantes 0.047 y el consumo de corriente eléctrica fue de 0.000 . Finalmente, el ingreso económico dio como resultado 0.039 , en el cual comparando con el 0.05 que es el parámetro estadísticamente aceptado, se puede determinar que existe regresión entre las variables; por lo anterior la hipótesis general se verifica para esta investigación.
Adicionalmente se realizaron tres correlaciones con los valores determinados en terreno para las variables intervinentes. La variable dependiente Y: es la generación de residuos sólidos y las variables independientes x 1: ingreso económico, $\mathrm{x} 2$ : gasto del consumo de energía y $\mathrm{x} 3$ : número de habitantes. Las ecuaciones de las curvas de correlación determinadas se muestran en la Tabla.

Tabla 7. Ecuaciones de las líneas de tendencia al correlacionar variables

\begin{tabular}{llll}
\hline Descripción & $y \rightarrow x 1$ & $y \rightarrow x 2$ & $y \rightarrow x 3$ \\
\hline Modelo Logarítmico & $\mathrm{y}=0.2478 \ln (\mathrm{x})-0.9611$ & $\mathrm{y}=0.2482 \ln (\mathrm{x})-0.2232$ & \\
& $R_{-.}^{2}=28$ & $R^{2}=14$ & \\
& $\mathrm{R}=0.4927$ & $\mathrm{R}=0.6491$ & \\
\hline \multirow{2}{*}{ Modelo Polinomial } & $\mathrm{y}=-0.0000002 \times 2+0.0007 \mathrm{X}+0.2586$ & $\mathrm{y}=-0.00002 \times 2+0.0081 \mathrm{x}+0.3757$ & $\mathrm{y}=$ \\
& $\mathrm{R} 2=0.2615$ & $\mathrm{R} 2=0.5146$ & $0.0148 \times 2-0.198 \mathrm{x}+1.24$ \\
& & & $\mathrm{R} 2=0.1659$ \\
& $\mathrm{R}=0.5113$ & $\mathrm{R}=0.7174$ & $\mathrm{R}=0.4073$ \\
\hline \multirow{2}{*}{ Modelo Exponencial } & $\mathrm{y}=0.0599 \mathrm{x}(0,3577)$ & $\mathrm{y}=0.1786 \mathrm{x}(0,3508)$ & \\
& $\mathrm{R} 2=0.2547$ & $\mathrm{R} 2=0.5051$ & \\
& $\mathrm{R}=0.5046$ & $\mathrm{R}=0.7107$ & \\
\hline
\end{tabular}

La correlación entre la producción de residuos sólidos y el consumo de electricidad de los hogares muestreados, presenta valores entre 0.6491 y 0.7174 . El modelo Polonomial de segundo grado, describe mejor la relación entre las dos variables mencionadas, su coeficiente de correlación $\mathrm{R}=0.7174$, se interpreta como una correlación positiva fuerte.

Presenta un punto de inflexión para $X=203$, se obtuvo al igualar a cero la primera derivada de su ecuación generatriz. Interpretando este valor de la siguiente manera: la generación de residuo sólido aumenta sostenidamente según aumenta el consumo de energía eléctrica, pero tiende a estabilizarse a partir cuando el consumo es de $\mathrm{X}=$ 210 soles/mes. Para consumos superiores a este valor, se producen incrementos menores en los residuos sólidos.

Por otro lado, al correlacionar las variables generación de residuos e ingreso económico mensual bruto, se nota que el rango donde se ubica el coeficiente de correlación es mayor que la anterior [0.4927 - 0.5113]. El modelo que mejor representa esta correlación es de tipo Polinomial con un coeficiente de correlación $\mathrm{R}=$
0.5113 , que se interpreta como correlación positiva considerable.

En este modelo se determinó el punto de inflexión para un valor de 1750 soles, lo que indica que cuanto mayor es el ingreso económico, mayor es la generación de residuos sólidos. Esta relación tiende a estabilizarse a partir de 1750 soles/mes.

\section{CONCLUSIONES}

A través de un modelo matemático de regresión múltiple, se obtiene una estimación confiable de la generación de residuos sólidos municipales.

Se determina la generación de RSU distrito de huacho, en forma factible y económica, aplicando el Modelo Matemático Polinomial de segundo grado, su coeficiente de correlación $\mathrm{R}=$ 0.511 entre las variables socioeconómicas y la generación de RSU. Con 95\% de confianza de relación significativa entre el ingreso económico y la GRS $(\mathrm{r}=.658 ; \mathrm{p}=0.00)$, el número de habitantes y la GRS $(r=.337 ; p=0.000)$, y el consumo de energía eléctrica y la GRS $(\mathrm{r}=.487$; $\mathrm{p}=0.00)$. Se determina, mayor ingreso económico mayor será la GRS. 


\section{REFERENCIA BIBLIOGRÁFICA}

Arnillas L., F., Merino G., M., Sueiro C., J. y Suárez M., M. (2009). El manejo de los residuos sólidos en los distritos costeros del norte chico del departamento de Lima (Barranca, Huaral y Huaura). Primera Edición. Perú: NOVAPRINT SAC.

Ávila Acosta, R.B. (2010). Estadística Elemental. Estudio y Ediciones RA. Lima, Perú.

Blas B., J. (2007). Vigilancia de la gestión de los Residuos Sólidos Municipales. Hospital General de Huacho, Área de Salud Ambiental. Huacho, Perú.

Brunal B., Ch., Giraldo G., E. y Caicedo, B. (2005). Calibración de un modelo matemático aplicado en rellenos sanitarios. De partame nto de Ingeniería civil y Ambiental. Universidad de los Andes. Bogotá, Colombia.

CONAM (2005). Premio anual a la gestión responsable en el manejo de los Residuos Sólidos. [En línea] 2008. [Fecha de acceso 08 de abril del 2008].

Chung P., A. (2002), Análisis económico de la ampliación de la cobertura del manejo de Residuos por medio de la Segregación en la Fuente en Lima Cercado. Tesis. Universidad Nacional mayor de San Marcos. Lima, Perú.

Cruz UFM. (2000). Fundamentos científicos de los modelos. México.

Del Val,A. (1997). El Tratamiento de los Residuos Sólidos Urbanos. Ministerio de Fomento de España. [En línea] 2009. [Fecha de acceso 10 de marzo del 2009]. URL disponible en: http://habitat.aq.upm.es/cs/ p3/a014.html

Digesa (1996). Relleno Sanitario Manual. Dirección General de Salud Ambiental del Ministerio de Salud. Lima, Perú.

Espinace, R. y Palma, J. (1983). Relleno Sanitario y su empleo como suelo de Fundación. Universidad Católica de Valparaíso. Chile:AIDIS

Flores, D. y Villafuerte, I. (2003). Guía práctica $\mathrm{N}^{\mathrm{o}} 1$ para la realización de estudios de $\mathrm{r}$ e s idu os só $1 \mathrm{idos}$ domiciliarios en ciudades. Programa de Gestión Urbana de las Naciones

Unidas. IPES. Promoción del desarrollo sostenible. Quito, Ecuador.
Glynn, Henry y GARY, Heinke. ( 1999 ). Ingeniería Ambiental. Segunda Edición. Editorial PrenticeHall. México.

Gonzales R., José T. (1996). Contaminación Urbana, Participación Ciudadana, Gobiernos Locales y Regionales. Editorial Saywa. Lima, Perú.

Gutiérrez C., Percy y Espinoza L., Oscar. (1996). Guía Técnica para el reciclaje de residuos plásticos. Instituto de Promoción de La Economía Social, IPES. Lima, Perú.

Hernández R. S., Fernández. C. y Baptista L. P. (2006). Metodología de la investigación. Cuarta Edición. Mc Graw-Hill Interamericana. México.

Hilario R., J. (2004). Gestión de Tratamiento de Residuos Sólidos y su Influencia en la Conservación del medio Ambiente y el Ecosistema en la Provincia de Huánuco. Tesis para optar el Grado de Magister en Ingeniería Industrial. Universidad $\mathrm{Nacional} \mathrm{Herminio}$ Valdizan. Huánuco, Perú.17. 